\title{
Effect of Bifurcation Angle Configuration and Ratio of Daughter Diameters on Hemodynamics of Bifurcation Aneurysms
}

\author{
A. Farnoush, A. Avolio, and Y. Qian
}

\begin{abstract}
BACKGROUND AND PURPOSE: EL associated with ruptured aneurysms is higher than that for unruptured aneurysms. In this study, the effect of arterial morphologic variation of bifurcation aneurysms on EL was investigated in idealized models of middle cerebral artery aneurysms.
\end{abstract}

\begin{abstract}
MATERIALS AND METHODS: Bifurcation angle configuration and DA ratio were evaluated in 6 idealized numeric models. Type A and B bifurcation models were defined with symmetric and asymmetric bifurcation angles of $136^{\circ}$, and $57^{\circ}$ and $79^{\circ}$, respectively. Three models with DA ratios of 1, 1.3, and 2 were constructed for each type. EL was calculated as the energy difference between aneurysm inflow and outflow at the aneurysm neck. Three growth paths (R1, R2, and R3) were proposed.

RESULTS: The highest EL and influx occurred in bifurcations with DA ratios of 1 for both types A and B. As the DA ratio increases, flow distribution between branches becomes more asymmetric, resulting in a reduction of EL and intra-aneurysmal flow. No strong relation was found between bifurcation angle configuration, inflow flux, and EL. EL decreased with an increase in the AR and DA ratio and increased with an increase in the AR and reduction of DA ratio.
\end{abstract}

CONCLUSIONS: EL determined in idealized models is less dependent on bifurcation angle configuration than on DA ratio, and the stability of the aneurysm strongly depends on variation of the daughter artery morphology after aneurysm growth.

ABBREVIATIONS: $\mathrm{AR}=$ aspect ratio; $\mathrm{DA}=$ daughter artery diameter; $\mathrm{EL}=$ energy loss; $\mathrm{LSA}=$ low shear stress area; $\mathrm{R} 1=$ increasing the $\mathrm{AR}$ and $\mathrm{DA}$ ratio; $\mathrm{R} 2=$ increasing the $A R$ and reducing the DA ratio; $R 3=$ increasing the AR with a constant DA ratio; WSS = wall shear stress

$\mathrm{H}$ emodynamics is recognized as having an important role in cerebral aneurysm formation, development, and rupture. Computational hemodynamic technologies have been used extensively to explain these mechanisms by using vascular structures from patient-specific $3 \mathrm{D}$ angiography. ${ }^{1-4}$ To date on the

\footnotetext{
Received February 22, 2012; accepted after revision May 21.

From the Australian School of Advanced Medicine, Macquarie University, Sydney, Australia.

Author contributions to the study and manuscript preparation include the follow ing: conception and design-A. Farnoush, A. Avolio, and Y. Qian; acquisition of data-A. Farnoush and Y. Qian; analysis and interpretation of data-A. Farnoush; drafting the article-A. Farnoush; critically revising the article-A. Avolio and $\mathrm{Y}$. Qian; reviewing the final version of the manuscript and approving it for submission—all authors; study supervision-A. Avolio and Y. Qian.

This work was supported by the Australian Research Council Linkage Grant LP0990263. Azadeh Farnoush was supported by Macquarie University Research Excellence Scholarship.

Paper previously presented in part in abstract form at: 11th Congress of the World Federation of Interventional and Therapeutic Neuroradiology, November 8-11, 2011; Cape Town, South Africa.

Please address correspondence to: Yi Qian, PhD, Australian School of Advanced Medicine, Macquarie University, Sydney, Australia; e-mail: yi.qian@mq.edu.au

- Indicates open access to non-subscribers at www.ajnr.org

http://dx.doi.org/10.3174/ajnr.A3222
}

basis of current hemodynamic parameters, no method has been found to be effective at predicting the risk of aneurysm growth and rupture. Thus, investigating additional parameters would be necessary.

Various types of aneurysm morphology simulations have been used for assessing the role of morphology in predicting the risk of rupture. ${ }^{5,6}$ By improving the generation of idealized bifurcation models, ${ }^{7}$ classifying the saccular intracranial aneurysm, ${ }^{8}$ and conducting in vitro analysis of bifurcation aneurysms, simulation methods become more effective. ${ }^{9,10}$ However, a study on the effect of hemodynamic parameters such as WSS in 20 MCA aneurysms has suggested the pathogenic effect of high WSS in the initiation of aneurysms and of low WSS in the growth and rupture of cerebral aneurysms. ${ }^{11}$ Any changes in intra-aneurysmal flow due to the appearance of a vortex and stagnation affect the EL of the entire system. Thus, calculating EL at the neck on the basis of the difference between energy transport to the aneurysm by inflow and energy loss by the outflow can provide information about the system and represents the power of collision applied by hemodynamic forces. Because this concept has already been used by cardiovascular surgeons to evaluate the outcome of surgery, 


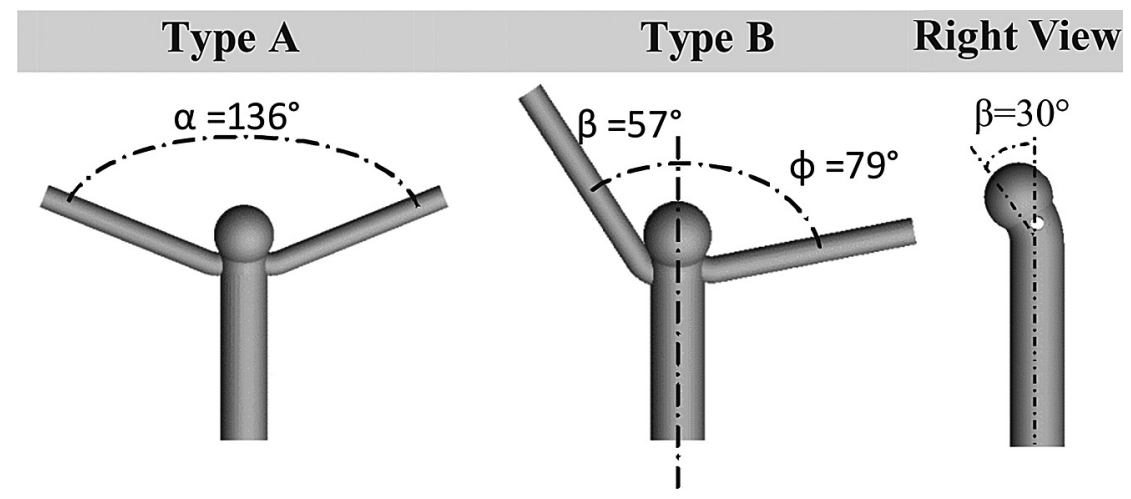

FIG 1. Idealized bifurcation aneurysm models at DA ratio $=1$. Types $A$ and $B$ represent symmetric and asymmetric bifurcation angles of $\alpha=136^{\circ}, \beta=57^{\circ}, \varphi=79^{\circ}$ respectively. The right side view shows $30^{\circ}$ between the aneurysm and parent artery.

Table 1: Configuration of 3 proposed growth paths of R1, R2, and R3 in bifurcation aneurysms ${ }^{a}$

\begin{tabular}{llll}
\hline & AR $=1.1$ & AR $=1.6$ & AR $=2.1$ \\
\hline R1 & DA ratio $=1$ & DA ratio $=1.3$ & DA ratio $=2$ \\
R2 & DA ratio $=2$ & DA ratio $=1.3$ & DA ratio $=1$ \\
R3 & DA ratio $=1$ & DA ratio $=1$ & DA ratio $=1$ \\
\hline
\end{tabular}

a Flow rate was considered constant during the growth paths.

it was assumed that EL can be used to explain the growth of aneurysms. A further study concentrating on EL of 4 ruptured and 26 unruptured ICA-posterior communicating artery aneurysms found that the EL of ruptured cases was 5 times higher than that of unruptured cases. $^{12}$

Moreover, a study of 210 cerebral aneurysms showed the presence of a higher kinetic energy ratio in ruptured aneurysms compared with unruptured ones. ${ }^{13}$ A study of 26 aneurysms also indicated that a larger region of low WSS was associated with ruptured aneurysms. ${ }^{14}$

Because morphologic analysis and hemodynamic parameters alone could not adequately explain why aneurysms develop or rupture at the apex of bifurcations, novel approaches were used to investigate the role of both morphology and hemodynamic parameters on aneurysm rupture. ${ }^{15} \mathrm{~A}$ previous study of 44 unruptured aneurysms selected from 1612 patients showed that aneurysms located at bifurcations, especially the anterior communicating artery and the middle cerebral artery, bleed easily compared with those located at the branching and bending points. ${ }^{16}$ Therefore, due to the high sensitivity of bifurcation aneurysms to variations of flow pattern and morphology, the hemodynamic parameter EL was used to evaluate the growth and rupture of this type of aneurysm. The aim of this study was to elucidate the relationship between the morphologic parameters of bifurcation aneurysms, including the bifurcation angle configuration; DA ratio; and hemodynamic parameters of EL and abnormal low WSS. Because of the high risk of rupture, not enough information is available for the study of long-term follow-up of cerebral aneurysm morphologies. In a study of $22 \mathrm{MCA}$ aneurysms, 7 cases were found to be located at the midline (4 ruptured and 3 unruptured). ${ }^{17}$ Among those aneurysms, only 1 ruptured aneurysm had symmetric bifurcation angles. Therefore, the present study addresses the effect of bifurcation configuration on EL and low WSS associated with rupture. In addition, 3 possible growth paths based on 3 discrete combinations of aspect ratios and DA ratios were investigated to elucidate the relation between aneurysm evolution and daughter artery morphology.

\section{MATERIALS AND METHODS Idealized Bifurcation Aneurysm Model}

Symmetric and asymmetric numeric phantoms of bifurcation aneurysms were created to evaluate the effect of bifurcation configuration on hemodynamic parameters. Values of bifurcation angles for idealized models were determined from an MCA bifurcation ruptured aneurysm of a male patient, 51 years of age. The bifurcation angles of this patient located at the midline of the parent artery were measured as $57^{\circ}$ and $79^{\circ} .{ }^{17}$ In the present study, we considered 2 types of bifurcation models: a symmetric bifurcation angle of $136^{\circ}$ (type A) and a bifurcation angle set asymmetrically at $57^{\circ}$ and $79^{\circ}$ in reference to the midline (type B, Fig 1). Parent artery and branch geometries were selected as described in the clinical literature with a proximal inlet diameter of $4.0 \mathrm{~mm}$ and both distal outlet diameters of $2.0 \mathrm{~mm} .{ }^{18}$ The size of the bifurcation aneurysm was reported as $4.3 \mathrm{~mm} .{ }^{17}$ Although the size of this aneurysm was not considered in the large aneurysm category with higher risk of rupture, ${ }^{19}$ it was reported as ruptured. Hence, a spheric aneurysm shape with a size of $4.2 \mathrm{~mm}$ and a neck width of $4 \mathrm{~mm}$ was set for both models. Previous studies have shown that the flow pattern in bifurcation aneurysms, in which the aneurysms have zero degree relative to the parent artery with equal flow-division outlets, was highly unstable ${ }^{10}$ and very sensitive to the type of blood flow model (Newtonian versus non-Newtonian). ${ }^{20}$ In addition, measurement of the aneurysm angle relative to the parent artery in our patient-specific clinical data base for the MCA showed that this angle varied between $30^{\circ}$ and $60^{\circ}$. Hence, the angle of the aneurysm dome relative to the parent artery was considered $30^{\circ}$ in this study (Fig 1). The DA ratio was defined as the ratio of the diameter of the larger branch to the diameter of the smaller branch. Models A-I, A-II, and A-III with symmetric angles of $136^{\circ}$ were constructed with DA ratios of 1 , 1.3 , and 2, respectively. Models B-I, B-II, and B-III with asymmetric angles of $57^{\circ}$ and $79^{\circ}$ were also created with the same DA ratios.

A straight entry tube of 20 times the parent artery diameter was attached to the inlet to remove inlet effects on the computed flow field and to ensure a fully developed boundary layer at the region of entry. ${ }^{21}$ The outlet boundary was located 50 times the branch artery diameter from the bifurcation to eliminate the effect of boundary condition locations on the flow inside the artery. ${ }^{22}$ Aneurysm growth was simulated by constructing 3 spheric-shaped aneurysms of $4.2,6.3$, and $8.2 \mathrm{~mm}$. The neck width was $4 \mathrm{~mm}$ for all models. Hence, the AR (aneurysm size/neck width) of the aneurysm was 1.1, 1.6 and 2.1, respectively. Three possible growth paths were investigated as described in Table 1. 
Type A

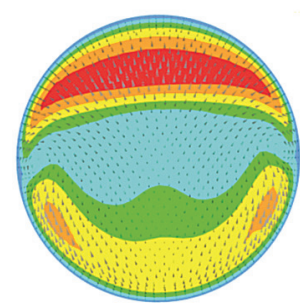

A-I
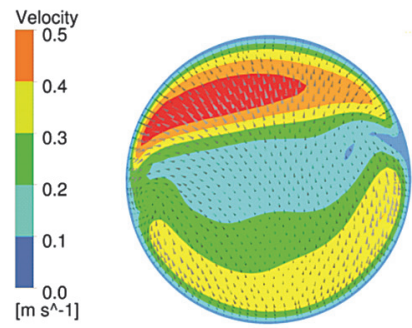

A-II

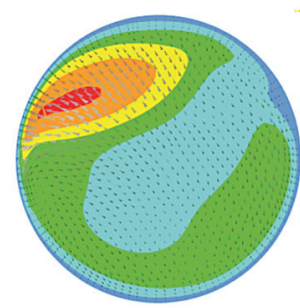

A-III
Type B

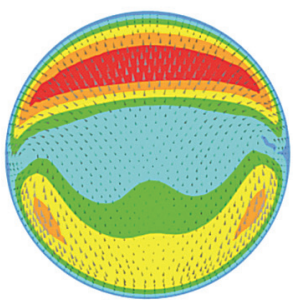

B-I

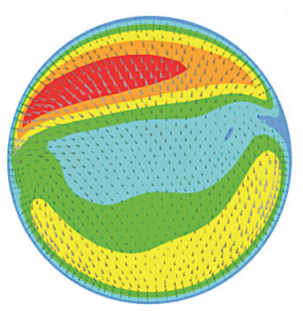

B-II

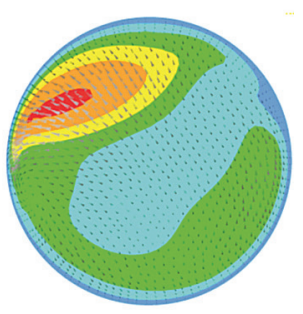

B-III
FIG 2. Velocity profile at the aneurysm neck for type $A$ and $B$ models. Increasing the DA ratio causes the flow distribution to become asymmetric, and less flow enters the aneurysm, as shown in A-III and B-III.

\section{Blood Flow Modeling and Boundary Conditions}

The major purpose of this study is to estimate the difference between various bifurcation angle configurations and aneurysm sizes. Our previous study indicated that there is no significant difference between the average volume of pulsatile flow and steady flow. ${ }^{12}$ Thus in this study, calculations were performed with a steady flow rate $(218 \mathrm{~mL} / \mathrm{min})$ at the parent artery inlet for idealized models by using the peak flow rate in the MCA. ${ }^{23}$ To characterize the flow regimen in the parent artery and branches, we calculated the Reynolds number, indicating the occurrence of laminar blood flow in all models (at the inlet and outlet, the Reynolds number based on maximum velocity was 340 and 360, respectively). Secondary flow at the inlet zone would undoubtedly influence the intra-aneurysm hemodynamics. However, at similar levels of Reynolds number, the effect of secondary flow on results is relatively negligible. In this study, an incompressible steady-state laminar flow model was considered for the simulation. In large arteries (diameter $>0.5 \mathrm{~mm}$ ), the non-Newtonian behavior of the blood flow is negligible, so blood was assumed to be a Newtonian fluid ${ }^{24}$ with blood flow density and dynamic viscosity of $1050 \mathrm{~kg} / \mathrm{m}^{3}$ and $0.0035 \mathrm{~Pa} \cdot \mathrm{s}$, respectively. ${ }^{7}$ By adopting the conventional assumption of lack of resistance in the cerebral circulation, we set the outlet boundary conditions to zero pressure. ${ }^{25}$ Along the artery and aneurysm wall, a no-slip flow bound-
Table 2: Comparison of flow distribution between branches at different DA ratios ${ }^{a}$

\begin{tabular}{|c|c|c|c|c|c|c|}
\hline & \multicolumn{2}{|c|}{$\mathrm{DA}=1$} & \multicolumn{2}{|c|}{$\mathrm{DA}=1.3$} & \multicolumn{2}{|c|}{$D A=2$} \\
\hline & $\begin{array}{c}\text { Larger } \\
\text { Branch } \\
(\%) \\
\end{array}$ & $\begin{array}{c}\text { Smaller } \\
\text { Branch } \\
(\%)\end{array}$ & $\begin{array}{c}\text { Larger } \\
\text { Branch } \\
(\%)\end{array}$ & $\begin{array}{c}\text { Smaller } \\
\text { Branch } \\
(\%)\end{array}$ & $\begin{array}{c}\text { Larger } \\
\text { Branch } \\
(\%)\end{array}$ & $\begin{array}{c}\text { Smaller } \\
\text { Branch } \\
(\%) \\
\end{array}$ \\
\hline Type & 50 & 50 & 72 & 28 & 91 & 9 \\
\hline Type B & 50 & 50 & 72 & 28 & 91 & 9 \\
\hline
\end{tabular}

${ }^{a}$ The results show that the larger the DA ratio is, the more difference there is between flow rates at branches.

ary condition was imposed at the inner lumen of the vessel, and arteries were assumed to be rigid.

\section{Computational Tools}

ICEM 13.0 (ANSYS, Lebanon, New Hampshire) was used for grid generation. The models were constructed with unstructured tetrahedral and prismatic mesh. The grid independency test showed that when the grid number was increased to $1.5 \times 10^{6}$, the EL at the aneurysm neck converged to a constant value. To increase the accuracy of WSS calculation near wall regions, we generated 5 prismatic layers with an average nodal space increasing by a ratio of 1.2 where the distance of the first layer to the vessel was fixed at $0.01 \mathrm{~mm}$. The CFD simulations were performed by using commercial finite-volume software (CFX.13.0; ANSYS) to analyze the flow model.

\section{Energy Loss Calculation}

Energy loss due to the presence of the aneurysm at the apex of the bifurcation can be calculated as follows:

$$
\begin{gathered}
\text { Energy loss }(\mathrm{EL})=E_{\text {inlet }}-E_{\text {outlet }} \\
E L=\frac{\sum\left(P_{i}+\rho \frac{1}{2} \nu_{i}^{2}\right) Q_{i}}{E_{\text {inlet }}}-\frac{\sum\left(P_{o}+\rho \frac{1}{2} \nu_{o}^{2}\right)}{E_{\text {outlet }}} Q_{o}
\end{gathered}
$$

where $P$ and $v$ are the static pressure and velocity, respectively; $i$ indicates the inflow to the aneurysm through aneurysm neck; and $o$, the outflow of the aneurysm through the aneurysm neck. $E_{\text {inlet }}$ and $E_{\text {outlet }}$ are the spatially averaged energy values over the crosssection of the neck. Dependency of the energy loss on the pressure and velocity of the blood flow entering the aneurysm as shown in equation 2 indicates that this parameter is strongly dependent on geometry.

WSS varies in the aneurysm and arteries due to differences in size. Therefore, the mean WSS of the parent artery was assumed as a reference value to compare with the WSS of the aneurysm. The abnormally low WSS region $\left(A_{1}\right)$ was calculated as an area of the aneurysm with a WSS smaller than $10 \%$ of the reference value. ${ }^{14}$ LSA quantifies the region of the aneurysm that is exposed to the abnormally low WSS and can be defined as a percentage of the

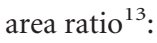

$$
\text { LSA }=100 \times\left(A_{1} / A_{a}\right)
$$

where $A_{\mathrm{a}}$ is the aneurysm sac area.

\section{RESULTS}

Effect of Bifurcation Angle and DA Ratio on Flow Pattern

Figure 2 shows the velocity profiles at the aneurysm neck of type A 
and $\mathrm{B}$ models, in which the main flow from the parent artery divides into 3 sections. Two portions of the blood flow enter directly into the branches. The third goes to the aneurysm from the anterior side of the neck and exits from the posterior side to the branches for both models. Red represents the inflow velocity; yellow in A-I, A-II, B-I, and B-II; and green in A-III and B-III represent the outflow regions. The outflow regions were $43 \%$ larger than the inflow regions for all models ( 8.1 versus $5.6 \mathrm{~mm}^{2}$ ) except for A-III and B-III, which increased to $48 \%$. Calculation of the influx magnitude into the aneurysm indicated that the intra-aneurysmal flow does not depend on bifurcation angle configuration. However, increasing the DA ratio from 1 to 2 for both types $\mathrm{A}$ and $\mathrm{B}$ changed the blood flow distribution between branches as shown in Table 2. The results show that with the larger DA ratio, the difference is greater between flow rates of branches. Therefore, the influx magnitude into the aneurysm reduced 31\% from A-I and B-I to A-III and B-III, respectively.

\section{Effect of Bifurcation Angle and DA Ratio on EL and WSS}

Energy loss at the aneurysm neck due to circulation of the blood inside the aneurysm was calculated for types A and B. As shown in Fig $3 A$, similar to flow patterns, a strong association is not found between the configuration of the bifurcation angle (symmetric and asymmetric) and the EL, though the EL difference increases to $4.3 \%$ for larger DA ratios. However, asymmetric flow distribution between branches leads to a larger portion of the main flow to the larger branches. Due to reduction of inflow to the aneurysm, the EL decreased by $38 \%$ from A-I to A-III and by $34.5 \%$ from B-I to B-III (Fig 3B).

LSA was calculated for all scenarios. The results demonstrated that for EL, there is only a 3.7\% difference between the LSA of types A and B (2.15\% for A-I and 2.23\% for Type B-I). However, a $95 \%$ difference in LSA was found as the DA ratio increased from 1 to 2 for both types of A and B models (Fig 3C).

\section{Effect of Aneurysm Growth on Daughter Artery Bifurcation}

The potential of numeric simulation in creating an idealized model based on patient-specific images is being increasingly recognized. ${ }^{26}$ In the present study, we propose 3 possible growth paths: growth path R1, growth path R2, and growth path R3. The EL of bifurcation aneurysms was calculated for growth paths R1, $\mathrm{R} 2$, and R3 as shown in Fig 4. The results indicated that if the aneurysm grows according to growth path $\mathrm{R} 1$, there is a reduction of $24 \%$ in $\mathrm{EL}$ from $\mathrm{AR}=1.1$ and $\mathrm{DA}$ ratio $=1$ to $\mathrm{AR}=2.1$ and $\mathrm{DA}$ ratio $=2$. In contrast, development of the aneurysm based on growth path R2 showed a 32\% increase of EL from AR $=1.1$ and $\mathrm{DA}$ ratio $=2$ to $\mathrm{AR}=1.6$ and $\mathrm{DA}$ ratio $=1$. Furthermore, growth path $\mathrm{R} 3$ showed only a reduction of $9.0 \%$ from $\mathrm{AR}=1.1$ to $\mathrm{AR}=$ 2.1 if there is no change in the morphology of the daughter arteries due to development of the aneurysm (DA ratio of 1 for all ARs).

A decrease of EL in growth paths R1 and R3 suggests that if the growth of the aneurysm is associated with an increase or constant DA ratio, the aneurysm may remain stable. However, if the growth of the aneurysm follows growth path R2 with an increase of EL as the DA ratio decreases, the intra-aneurysmal flow becomes unstable and the aneurysm may be at high risk. This result
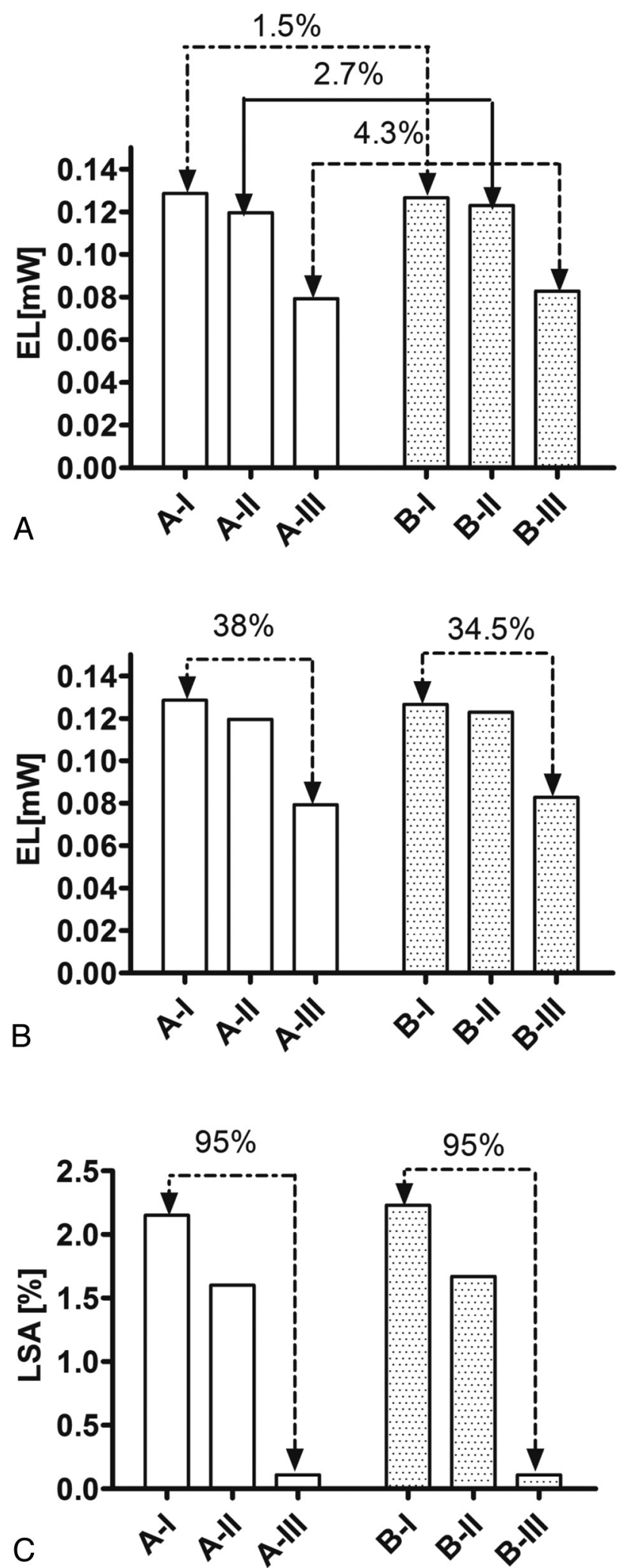

FIG 3. Comparison of EL calculated at the aneurysm neck of type $A$ and $B$ models based on bifurcation angle configuration $(A)$ and different DA ratios $(B)$. C, LSA calculation in both type $A$ and $B$ models at different $D A$ ratios.

is in agreement with our clinical observations from follow-up of MCA aneurysms on 3 sequential CT scans, demonstrating a variation of the DA ratio from 2.5 to 1.8 as the aneurysm grows. 


\section{DA ratio}

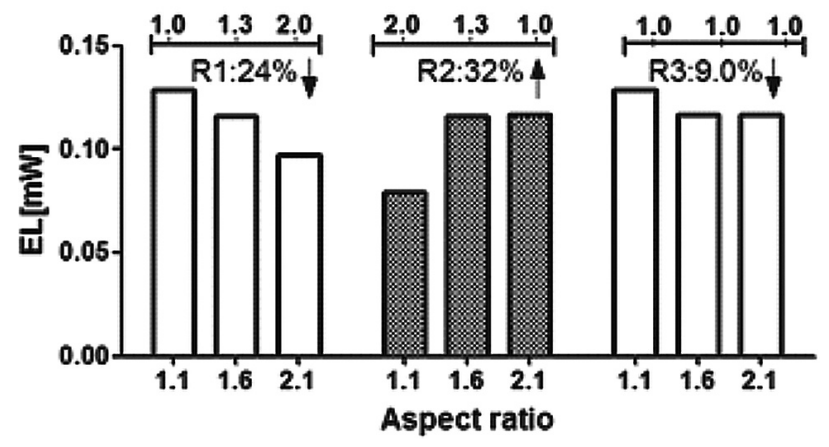

FIG 4. EL calculated for the proposed 3 growth paths of bifurcation aneurysms. In growth path $\mathrm{Rl}$, there is $24 \%$ reduction in EL by increasing the AR and DA ratio. In growth path R2, a 32\% increase can be seen in EL as the AR increases and the DA ratio decreases. Growth path R3 shows that if the DA ratio does not change during the aneurysm evolution, the $E L$ decreases by $9.0 \%$ from $A R=1.1$ to $A R=2.1$.

\section{DISCUSSION}

The flow pattern and energy loss of a bifurcation aneurysm at 2 types of bifurcation angle and a variety of DA ratios are presented in the current study. The results show that the DA ratio significantly affected the flow pattern and EL at the aneurysm neck for both types of bifurcation angle configurations. However, no strong correlation was found between this angle configuration and EL.

Our results are in agreement with the findings of Sadatomo et $\mathrm{al},{ }^{27}$ who measured the AR and DA ratio of 20 unruptured and 24 ruptured MCA aneurysms. Morphologic measurements identified a higher AR and a lower DA ratio among the ruptured compared with the unruptured groups (AR: $2.24 \pm 0.75$ for ruptured and $1.56 \pm 0.8$ for unruptured; DA: $1.53 \pm 0.54$ for ruptured and $2.14 \pm 0.8$ for unruptured). They concluded that even branch diameter and aneurysm neck located at the center of the bifurcation might be related to aneurysm rupture. The present results confirmed that the highest rate of inflow and EL occurs at a DA ratio of 1 , when the diameters of both branches are similar and symmetric flow distribution is observed between these branches (model A-I: $0.128 \mathrm{~mW}$ and B-I: $0.126 \mathrm{~mW}$ ). A similar pattern was found at a DA ratio of 1.3 , with a $6.9 \%$ difference between the EL of A-I and A-II and 2.9\% between B-I and B-II. The significance of the DA ratio decreases when the bifurcation angle is asymmetric. However, at a DA ratio of 2 , the flow distribution between branches changes to 90:10, which affects the influx inside the aneurysm and EL, accordingly. The reduction of the EL for a DA ratio of 2 suggested that with the more even branch diameter with a lower DA ratio, the risk of rupture was higher.

The effect of the morphology of the aneurysm and parent and daughter arteries on aneurysm growth is generally poorly understood due to lack of access to follow-up patient-specific models. Previous studies have investigated the effect of scaling the parent artery $^{28}$ or removing the bleb from the aneurysm ${ }^{29}$ on the growth pattern. In the present study, we found that as the bifurcation aneurysm grows, any change in morphology of the daughter artery affects intra-aneurysmal flow and EL. Considering 3 possible growth paths (R1, R2, and R3) suggested that measurement of daughter artery diameter during the follow-up of the aneurysm can provide useful information about the stability of the aneurysm. Further investigations in patient-specific follow-up models are needed to confirm these observations.

A previous study of 26 intracranial aneurysms reported that the characteristics of WSS distribution are more dependent on the morphology of the aneurysm than on the flow rate and waveforms. ${ }^{14}$ Thus, measuring LSA in a consistent way at a single time point will not affect the results of the current study. A study of 119 aneurysms (38 ruptured and 81 unruptured) showed that ruptured aneurysms had larger LSAs and lower WSS magnitudes than those in the unruptured group. ${ }^{15}$ Our results have shown that even daughter artery diameters are associated with larger EL and LSA. Although EL is a global parameter that can evaluate the loss of energy in the system due to the appearance of a vortex, stagnation and intra-aneurysmal variations and LSA only represent areas locally affected by stagnation flow. No strong association was found between bifurcation angle configuration and both hemodynamic parameters of EL and LSA.

Because there is no agreement regarding the relation between available hemodynamic parameters such as WSS and the growth or rupture of aneurysms, in this study, EL was introduced as a possible new parameter that may improve the current knowledge of the risk of rupture. Once the sensitivity of EL to morphologic variations becomes clear, the next step will be categorizing the patient-specific models to evaluate the current results.

\section{CONCLUSIONS}

Morphologic study of bifurcation aneurysms with different bifurcation angle configurations and DA ratios has shown that the EL of the aneurysm is mostly dependent on the DA ratio, whereas the flow distribution between daughter arteries is affected by the branch diameter. The highest EL for bifurcation aneurysms was calculated for the symmetric branch diameter with even flow distribution. The results of this study indicate that measurement of the daughter artery diameter of bifurcation aneurysms by CT or MR imaging can provide valuable information about the intraaneurysmal flow pattern, flow distribution, and EL, all of which may be useful in the prediction of the risk of aneurysm rupture. Simulation of 3 proposed growth paths for bifurcation aneurysm evolution demonstrated that the stability of the aneurysm strongly depends on variation of the daughter artery morphology after aneurysm growth. Further investigation is necessary to evaluate this result in larger clinical databases.

\section{ACKNOWLEDGMENTS}

We thank Yuichi Murayama, MD, Hiroyuki Takao, MD, Winston Chong, MD, and Michael K Morgan, MD, for their constructive comments and advice for this study.

\section{REFERENCES}

1. Steinman DA, Milner JS, Norley CJ, et al. Image-based computational simulation of flow dynamics in a giant intracranial aneurysm. AJNR Am J Neuroradiol 2003;24:559-66

2. Hassan T, Timofeev EV, Ezura M, et al. Hemodynamic analysis of an adult vein of Galen aneurysm malformation by use of 3D imagebased computational fluid dynamics. AJNR Am J Neuroradiol 2003; 24:1075-82

3. Jou L-D, Quick CM, Young WL, et al. Computational approach to AJNR Am J Neuroradiol 34:391-96 Feb 2013 www.ajnr.org 395 
quantifying hemodynamic forces in giant cerebral aneurysms. AJNR Am J Neuroradiol 2003;24:1804-10

4. Castro M. Computational modeling of cerebral aneurysms in arterial networks reconstructed from multiple 3D rotational angiography images. In: Proceedings of the International Society for Optics and Photonics, San Jose, California. January 16-20, 2005;233-44

5. Ma B, Harbaugh RE, Raghavan ML. Three-dimensional geometrical characterization of cerebral aneurysms. Ann Biomed Eng 2004;32: $264-73$

6. Raghavan ML, Ma B, Harbaugh RE. Quantified aneurysm shape and rupture risk. J Neurosurg 2005;102:355-62

7. Zakaria H, Robertson AM, Kerber CW. A parametric model for studies of flow in arterial bifurcations. Ann Biomed Eng 2008;36:1515-30

8. Hassan T, Timofeev EV, Saito T, et al. A proposed parent vessel geometry-based categorization of saccular intracranial aneurysms: computational flow dynamics analysis of the risk factors for lesion rupture. J Neurosurg 2005;103:662-80

9. Liou TM, Chang TW, Chang WC. Pulsatile flow through a bifurcation with a cerebrovascular aneurysm. J Biomech Eng 1994;116: $112-18$

10. Steiger HJ, Poll A, Liepsch D, et al. Basic flow structure in saccular aneurysms: a flow visualization study. Heart Vessels 1987;3:55-65

11. Shojima M, Oshima M, Takagi $\mathrm{K}$, et al. Magnitude and role of wall shear stress on cerebral aneurysm: computational fluid dynamic study of $\mathbf{2 0}$ middle cerebral artery aneurysms. Stroke 2004;35: $2500-05$

12. Qian $\mathrm{Y}$, Takao H, Umezu M, et al. Risk analysis of unruptured aneurysms using computational fluid dynamics technology: preliminary results. AJNR Am J Neuroradiol 2011;32:1948-55

13. Cebral JR, Mut F, Weir J, et al. Quantitative characterization of the hemodynamic environment in ruptured and unruptured brain aneurysms. AJNR Am J Neuroradiol 2011;32:145-51

14. Jou LD, Lee DH, Morsi $\mathrm{H}$, et al. Wall shear stress on ruptured and unruptured intracranial aneurysms at the internal carotid artery. AJNR Am J Neuroradiol 2008;29:1761-67

15. Xiang J, Natarajan SK, Tremmel M, et al. Hemodynamic-morphologic discriminants for intracranial aneurysm rupture. Stroke 2011; 42:144-52

16. Ujiie $\mathrm{H}$, Sato $\mathrm{K}$, Ondo $\mathrm{H}$, et al. Clinical analysis of incidentally discovered unruptured aneurysms. Stroke 1993;24:1850-56
17. Sadatomo T, Yuki K, Migita K, et al. Evaluation of relation among aneurysmal neck, parent artery, and daughter arteries in middle cerebral artery aneurysms, by three-dimensional digital subtraction angiography. Neurosurg Rev 2005;28:196-200

18. Umansky F, Juarez SM, Dujovny M, et al. Microsurgical anatomy of the proximal segments of the middle cerebral artery. J Neurosurg 1984;61:458-67

19. Unruptured intracranial aneurysms-risk of rupture and risks of surgical intervention. $N$ Engl J Med 1998;339:1725

20. Valencia AA, Guzmán AM, Finol EA, et al. Blood flow dynamics in saccular aneurysm models of the basilar artery. J Biomech Eng 2006; $128: 516-26$

21. Jung J, Hassanein A, Lyczkowski RW. Hemodynamic computation using multiphase flow dynamics in a right coronary artery. Ann Biomed Eng 2006;34:393-407

22. Qian Y, Liu JL, Itatani K, et al. Computational hemodynamic analysis in congenital heart disease: simulation of the Norwood procedure. Ann Biomed Eng 2010;38:2302-13

23. Ford MD, Alperin N, Lee SH, et al. Characterization of volumetric flow rate waveformes in the normal internal carotid and vertebral arteries. Physiol Meas 2005;26:477-88

24. Hoi Y, Meng H, Woodward SH, et al. Effects of arterial geometry on aneurysm growth: three-dimensional computational fluid dynamics study. J Neurosurg 2004;101:676-81

25. Karmonik C, Klucznik R, Benndorf G. Comparison of velocity patterns in an AComA aneurysm measured with 2D phase contrast MRI and simulated with CFD. Technol Health Care 2008;16:119-28

26. Cebral JR, Castro MA, Appanaboyina S, et al. Efficient pipeline for image-based patient-specific analysis of cerebral aneurysm hemodynamics: technique and sensitivity. IEEE Trans Med Imaging 2005;24:457-67

27. Sadatomo T, Yuki K, Migita K, et al. Morphological differences between ruptured and unruptured cases in middle cerebral artery aneurysms. Neurosurgery 2008;62:602-09

28. Tremmel M, Dhar S, Levy EI, et al. Influence of intracranial aneurysm-to-parent vessel size ratio on hemodynamics and implication for rupture: results from a virtual experimental study. Neurosurgery 2009;64:622-30, discussion 630-31

29. Cebral J, Sheridan M. Hemodynamics and bleb formation in intracranial aneurysms. AJNR Am J Neuroradiol 2010;31:304-10 